\title{
Listeriosis Outbreaks Associated with Soft Cheeses, United States, 1998-2014 ${ }^{1}$
}

\author{
Kelly A. Jackson, L. Hannah Gould, \\ Jennifer C. Hunter, Zuzana Kucerova, \\ Brendan Jackson
}

Since 2006, the number of reported US listeriosis outbreaks associated with cheese made under unsanitary conditions has increased. Two-thirds were linked to Latin-style soft cheese, often affecting pregnant Hispanic women and their newborns. Adherence to pasteurization protocols and sanitation measures to avoid contamination after pasteurization can reduce future outbreaks.

$L$ isteria monocytogenes is a widely distributed environmental bacterium that can grow at refrigeration temperatures. Infection can cause severe illness and death. Persons at higher risk for infection include older adults, persons with weakened immune systems, and pregnant women and their newborns.

Listeriosis outbreaks have been associated with refrigerated ready-to-eat foods, including hot dogs, delicatessen meats, soft cheeses, milk, and other dairy products. For soft-ripened cheeses, the risk for listeriosis per serving is estimated to be 50 - to 160 -fold greater for cheese made from unpasteurized milk than pasteurized milk (1). Pasteurization kills L. monocytogenes; however, milk labeled as pasteurized and dairy products made from pasteurized milk can become contaminated due to inadequate hygiene practices after pasteurization. The earliest reported listeriosis outbreak in the United States in 1985, associated with Latin-style cheese (in particular, queso fresco and cotija), resulted in 142 illnesses, 28 deaths, and 20 fetal losses (2). Although the cheese was labeled as made from pasteurized milk, raw milk was inadvertently introduced into the pasteurized milk.

A US retail survey of several soft cheeses (Latin-style, blue-veined, mold-ripened) from 2000-2001 detected $L$. monocytogenes in $1.3 \%$ of cheeses made from unlabeled or unpasteurized milk and $0.5 \%$ of cheeses from pasteurized milk (3). However, pasteurized-milk cheese is much more commonly consumed than cheese made from unpasteurized milk. In a survey of food exposures conducted in 10 US states during 2006-2007, respondents reported eating

Author affiliation: Centers for Disease Control and Prevention, Atlanta, Georgia, USA

DOI: https://doi.org/10.3201/eid2406.171051 types of soft cheeses $(15.3 \%$ for blue-veined cheese, $6 \%-$ $11 \%$ for other soft cheeses; pasteurization status unknown) more frequently than they reported eating cheeses made from unpasteurized milk in the previous 7 days (1.6\%) (4). We describe outbreaks linked to soft cheese (both soft-ripened and acid-coagulated-ripened cheeses), demographic characteristics of the persons affected, and possible contributing factors to help inform prevention messaging for persons at higher risk.

\section{The Study}

Health departments in the United States electronically submit reports of foodborne disease outbreaks to the Foodborne Disease Outbreak Surveillance System (FDOSS). FDOSS captures information on etiology; implicated food; number of illnesses, hospitalizations, and deaths; and other features. We queried FDOSS for L. monocytogenes outbreaks ( $\geq 2$ cases) in the United States from 1998, when pulsed-field gel electrophoresis was first used to investigate listeriosis outbreaks, through 2014. We obtained information on fetal losses; deaths; number of cheese types; pasteurization status of milk used to make the cheese; recall issuance; and isolate subtyping from published reports (5-11), unpublished data, and food recall announcements. We considered infections in pregnant women or infants $\leq 28$ days of age to be pregnancy-associated. We considered outbreaks multistate if exposure to the implicated food occurred in $>1$ state.

Of 58 listeriosis outbreaks reported during 1998-2014, a total of $17(30 \%)$ were associated with soft cheese (Figure), and resulted in 180 illnesses, 14 fetal losses, and 17 deaths (online Technical Appendix Table, https://wwwnc. cdc.gov/EID/article/24/6/17-1051-Techapp1.pdf). Most patients $(146,88 \%)$ were hospitalized. Of 116 patients for whom we had information on ethnicity, 38 (33\%) were Hispanic. Of 140 cases with available data, 62 (44\%) were pregnancy-associated. Median outbreak size was 8 cases (range 2-34 cases). Ten outbreaks were multistate, and 16 were associated with commercial products, of which 14 involved cheeses produced in the United States. The proportion of listeriosis outbreaks linked to soft cheese made from pasteurized milk (12 outbreaks, 33\%) was significantly higher during 2007-2014 than during 1998-2006 (1 outbreak, 5\%;

\footnotetext{
${ }^{1}$ Preliminary results from this study were presented at the International Conference on Emerging Infectious Diseases; March 11-14, 2012; Atlanta, GA, USA.
} 


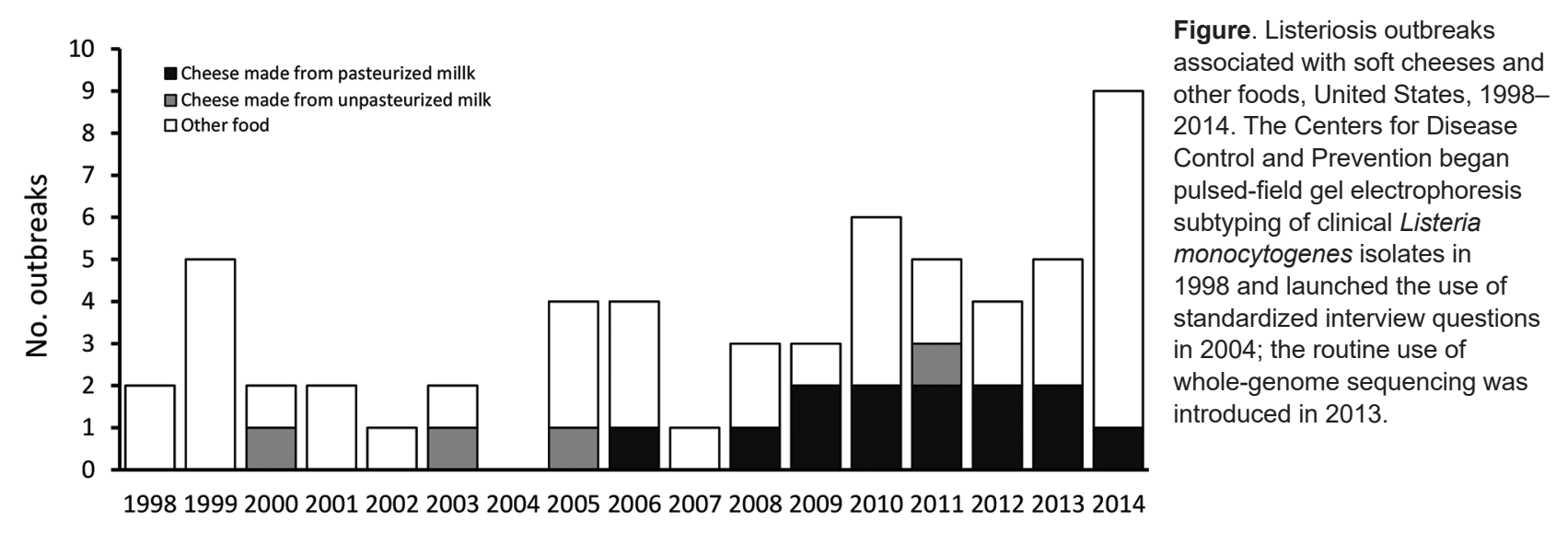

$\mathrm{p}=0.009)$. Clinical isolates from soft-cheese outbreaks predominantly fell in lineage I (14 outbreaks, $82 \%$ ). We found 2 sequence type (ST) and clonal complex (CC) combinations in multiple outbreaks (ST5/CC5, 5 outbreaks; ST6/ CC6, 2 outbreaks), whereas other ST and CC combinations appeared in single outbreaks (e.g., ST663 or ST558).

Latin-style cheeses were implicated in 11/17 (65\%) outbreaks, accounting for 98 (54\%) cases of listeriosis. The remaining outbreaks involved sheep's-milk cheese, Middle Eastern- or Eastern European-style cheeses, Italianstyle cheese, blue-veined cheese, and soft-ripened cheeses (1 outbreak each). Nearly all outbreaks (13/17) resulted in recalls.

FDA inspections of cheese-making facilities associated with outbreaks found sanitation and hygiene deficiencies (e.g., roof leaks over manufacturing equipment, an open sewer vent in a manufacturing room, and foodcontact aprons stored in restrooms) (7-9); pest infestations (e.g., cockroaches, flying insects) (8); failure to hold food at proper temperature $(8)$; and presence $(8,11,12)$ or persistence of $L$. monocytogenes in environmental niches of processing plants $(9)$.

\section{Conclusions}

Consumption of contaminated soft cheese made under unsanitary conditions continues to be a common cause of listeriosis outbreaks in the United States. Multiple types of soft cheeses have been implicated in outbreaks, with most outbreaks linked to Latin-style soft cheese. These outbreaks disproportionately affect Hispanic pregnant women and their neonates, a group with 24 times higher risk for listeriosis than that of the general US population (13). The proportion of listeriosis outbreaks caused by consumption of soft cheese made from pasteurized milk has increased in recent years. Reasons for the increase may include the growing US Hispanic population (which increased from $11 \%$ in 1998 to $17 \%$ in 2014 [14]); a 2.5 -fold increase in per capita consumption of cheese from 1980 to 2013 (15); consumer demand for certain types of cheeses; and an increase in the number of small producers, some of which had sanitary deficiencies. Better outbreak detection due to improved molecular subtyping and epidemiologic methods have resulted in a greater number of solved outbreaks; however, we did not observe a similar increased proportion of outbreaks linked to other foods during the same period. This finding suggests that changes in outbreak detection are unlikely to be the only contributor.

Despite the much higher risk for listeriosis per serving of cheese made from unpasteurized rather than pasteurized milk, during the study period, only about one quarter (4/17) of all outbreaks were linked to consumption of soft cheese made from unpasteurized milk. This result may be due, in part, to public health messages advising consumers at higher risk for listeriosis not to eat these cheeses.

For instances in which information was available, we noted environmental contamination and sanitation deficiencies in all outbreaks associated with cheese made from pasteurized milk. Although some of these deficiencies were unlikely to contaminate cheese directly, they indicate a lack of attention to sanitation and hygiene. This finding highlights the importance of robust sanitation and L. monocytogenes monitoring programs for cheese manufacturers.

Consumers, particularly persons at high risk for listeriosis, are advised to avoid unpasteurized milk and dairy products made from unpasteurized milk. Soft cheeses made with pasteurized milk, including commercial cottage cheese, cream cheese, and processed mozzarella, are generally considered safe. However, some soft cheeses made with pasteurized milk, particularly Latin-style soft cheeses, have been produced in facilities with improper processing conditions, resulting in L. monocytogenes contamination. Consumers cannot evaluate the conditions under which a cheese was made on the basis of labeling or other attributes of the product. We advise persons at higher risk for listeriosis (the elderly, persons with immunocompromising conditions, and pregnant women) to carefully consider whether to consume Latin-style and other soft cheeses implicated in previous outbreaks. 


\section{Acknowledgments}

We thank state, local, and territorial health departments for reporting outbreaks to FDOSS, and the CDC Enteric Diseases Laboratory Branch, Division of Foodborne, Waterborne, and Environmental Diseases, National Center for Emerging and Zoonotic Infectious Diseases, for providing isolate subtype data

\section{About the Author}

Ms. Jackson is an epidemiologist at the US Centers for Disease Control and Prevention. Her primary area of interest is infectious disease public health surveillance, with the objective of informing control measures.

\section{References}

1. US Food and Drug Administration and Health Canada. Joint FDA/ Health Canada quantitative assessment of the risk of listeriosis from soft-ripened cheese consumption in the United States and Canada: report. 2015 [cited 2016 March 3]. http://www.fda.gov/ downloads/Food/FoodScienceResearch/RiskSafetyAssessment/ UCM429419.pdf

2. Linnan MJ, Mascola L, Lou XD, Goulet V, May S, Salminen C, et al. Epidemic listeriosis associated with Mexican-style cheese. N Engl J Med. 1988;319:823-8. http://dx.doi.org/10.1056/ NEJM198809293191303

3. FoodRisk.org. Survey of Listeria monocytogenes in ready to eat foods. 2003 Mar [cited 2016 Apr 8]. http://foodrisk.org/exclusives/ SLMREF/

4. US Centers for Disease Control and Prevention. CDC, Foodborne Active Surveillance Network (FoodNet) population survey atlas of exposures. 2006-2007. Atlanta: US Department of Health and Human Services, Centers for Disease Control and Prevention; 2007 [cited 2018 Apr 16]. https://www.cdc.gov/foodnet/surveys/ foodnetexposureatlas0607_508.pdf

5. Centers for Disease Control and Prevention (CDC). Outbreak of listeriosis associated with homemade Mexican-style cheeseNorth Carolina, October 2000-January 2001. MMWR Morb Mortal Wkly Rep. 2001;50:560-2.

6. Jackson KA, Biggerstaff M, Tobin-D'Angelo M, Sweat D, Klos R, Nosari J, et al. Multistate outbreak of Listeria monocytogenes associated with Mexican-style cheese made from pasteurized milk among pregnant, Hispanic women. J Food Prot. 2011;74:949-53. http://dx.doi.org/10.4315/0362-028X.JFP-10-536

7. US Food and Drug Administration. FDA Form 483: Quesos Mi Pueblito, LLC. 2010 Jan 20 [cited 2016 Apr 8]. http://www.fda.gov/ ucm/groups/fdagov-public/@fdagov-afda-orgs/documents/ document/ucm198180.pdf

8. US Food and Drug Administration. Queseria Chipilo, Inc. 3/3/11 warning letter. 2011 Nov 8 [cited 2016 Apr 8]. http://fda-warningletters.blogspot.com/2011/03/queseria-chipilo-inc-3311.html

9. Acciari VA, Iannetti L, Gattuso A, Sonnessa M, Scavia G, Montagna C, et al. Tracing sources of Listeria contamination in traditional Italian cheese associated with a US outbreak: investigations in Italy. Epidemiol Infect. 2016;144:2719-27. http://dx.doi.org/10.1017/S095026881500254X

10. US Food and Drug Administration. FDA investigation summarymultistate outbreak of Listeria monocytogenes linked to certain Crave Brothers Farmstead Classics Cheeses. 2014 May 16 [cited 2016 Apr 11]. http://wayback.archive-it.org/ 7993/20171114154927/https://www.fda.gov/Food/ RecallsOutbreaksEmergencies/Outbreaks/ucm359588.htm

11. US Department of Justice. Delaware cheese company agrees to plead guilty to food adulteration charge, signs consent decree. 2016 Jan 29 [cited 2016 Apr 8]. https://www.justice.gov/opa/pr/ delaware-cheese-company-agrees-plead-guilty-food-adulterationcharge-signs-consent-decree

12. Michigan Department of Agriculture \& Rural Development Food and Dairy Division. Annual report fiscal year 2012 [cited $2016 \mathrm{Apr}$ 8]. https://www.michigan.gov/documents/mdard/FY12_MDARD_ Food and Dairy Annual report 419276 7.pdf

13. Silk BJ, Mahon BE, Griffin PM, Gould LH, Tauxe RV, Crim SM, et al.; Centers for Disease Control and Prevention (CDC). Vital signs: Listeria illnesses, deaths, and outbreaks - United States, 2009-2011. MMWR Morb Mortal Wkly Rep. 2013;62:448-52.

14. US Census Bureau. Hispanic origin data tables [cited 2017 Nov 20]. https://www.census.gov/topics/population/hispanic-origin/data/ tables.All.html.

15. US Department of Agriculture Economic Research Service. Food availability (per capita) data system. 2015 Nov 12 [cited 2016 Jul 6]. http://www.ers.usda.gov/data-products/food-availability-percapita-data-system/.aspx

Address for correspondence: Kelly A. Jackson, Centers for Disease

Control and Prevention, 1600 Clifton Rd NE, Mailstop A16, Atlanta, GA 30329-4027, USA; email: gqv8@cdc.gov 